\title{
AN UPPER BOUND FOR THE NUMBER OF ODD MULTIPERFECT NUMBERS
}

\author{
PINGZHI YUAN
}

(Received 21 September 2012; accepted 28 October 2012; first published online 28 January 2013)

\begin{abstract}
A natural number $n$ is called $k$-perfect if $\sigma(n)=k n$. In this paper, we show that for any integers $r \geq 2$ and $k \geq 2$, the number of odd $k$-perfect numbers $n$ with $\omega(n) \leq r$ is bounded by $\left(\begin{array}{c}\left\lfloor 4^{r} \log _{3} 2\right\rfloor+r \\ r\end{array}\right) \sum_{i=1}^{r}\left(\begin{array}{c}\lfloor k r / 2\rfloor \\ i\end{array}\right)$, which is less than $4^{r^{2}}$ when $r$ is large enough.

2010 Mathematics subject classification: primary 11A25.

Keywords and phrases: odd perfect numbers, $k$-perfect numbers.
\end{abstract}

\section{Introduction}

Let $k \geq 2$ be a positive integer. A natural number $N$ is said to be $k$-perfect (or multiperfect of abundancy $k$ ) if $\sigma(N)=k N$, where $\sigma(N)$ denotes the sum of all the divisors of $N$. We say $N$ is perfect when $k=2$. The even perfect numbers were completely classified by Euler. Namely, $N$ is an even perfect number if and only if $N=2^{p-1}\left(2^{p}-1\right)$, where $2^{p}-1$ is prime. However we know less about odd perfect numbers. We do not have a single example, and we do not have a proof that they do not exist.

Let $\omega(N)$ denote the number of distinct prime factors of a natural number $N$. In 1913, Dickson [4] proved that there are only finitely many odd perfect numbers with $k$ distinct prime factors. In 1977, Pomerance [8] gave an explicit upper bound in terms of $k$. Heath-Brown [5] improved the bound to $N<4^{4^{k}}$, and Cook [2] reduced this bound to $N<D^{4^{k}}$ with $D=(195)^{1 / 7}$. Nielsen [6] slightly improved and generalised Cook's method; he proved that if $N$ is an odd multiperfect number with $k$ distinct prime factors, then

$$
N<2^{4^{k}}
$$

In addition to an upper bound on the size of such $N$, Pollack [7] proved that for each positive integer $k$ the number of odd perfect numbers $N$ with $\omega(N) \leq k$ is bounded

Supported by NSF of China (No. 11271142) and the Guangdong Provincial Natural Science Foundation (No. S2012010009942).

(C) 2013 Australian Mathematical Publishing Association Inc. 0004-9727/2013 \$16.00 
by $4^{k^{2}}$. This result was generalised by Chen and Luo [1], who showed that, for any integer $r \geq 1$, the number of odd $k$-perfect numbers $n$ with $\omega(n) \leq r$ is bounded by $(k-1) \cdot 4^{r^{3}}$. More recently, Dai et al. [3] improved the bound of Chen and Luo to $4^{r^{2}}(k-1)^{2 r^{2}+3}$. The purpose of this paper is to improve the above result. We prove the following estimate.

THEOREM 1.1. For any integers $r \geq 2$ and $k \geq 2$, the number of odd $k$-perfect numbers $n$ with $\omega(n) \leq r$ is bounded by $\left(\begin{array}{c}4^{r}\left\lfloor\log _{3} 2\right\rfloor+r \\ r\end{array}\right) \sum_{i=1}^{r}\left(\begin{array}{c}\lfloor k r / 2\rfloor \\ i\end{array}\right)$, which is less than $4^{r^{2}}$ when $r$ is large enough.

\section{The proof}

The proof is essentially in the spirit of Pollack's work [7], and is a modification of Wirsing's method [9], but with a different counting argument. Let $x$ be a positive real number. Suppose that $N<x$ is an odd $k$-perfect number and $\omega(N) \leq r$. Write $N=A B$, where $A:=\prod_{p^{e} \| N, p>k r} p^{e}$ and $B:=\prod_{p^{e} \| N, p \leq k r} p^{e}$. We have

$$
\frac{\sigma(A)}{A}=\prod_{p^{e} \| A}\left(1+\frac{1}{p}+\cdots+\frac{1}{p^{e}}\right)<\prod_{p \mid A}\left(1+\frac{1}{p}+\frac{1}{p^{2}}+\cdots\right),
$$

and so

$$
\frac{A}{\sigma(A)}>\prod_{p \mid A}\left(1-\frac{1}{p}\right) \geq 1-\sum_{p \mid A} \frac{1}{p} \geq 1-\frac{r}{k r+1}>\frac{k-1}{k},
$$

which implies that $B>1$. Since $N$ is $k$-perfect, $\sigma(A B)=k A B$, and hence

$$
(k-1) B=\frac{k-1}{k} k B<\frac{A}{\sigma(A)} k B=\sigma(B) \leq k B,
$$

with equality on the right precisely when $A=1$. Suppose $A \neq 1$. By the previous inequality,

$$
\sigma(B)>(k-1) B \text { and } \sigma(B) \mid k A B .
$$

If $\operatorname{gcd}(A, \sigma(B))=1$, then by the second formula of (2.3), $\sigma(B) \mid k B$, and so $\sigma(B) \leq$ $k B / 2 \leq(k-1) B$, which contradicts (2.3). Therefore, there is a prime $p$ dividing $\operatorname{gcd}(A, \sigma(B))$, which means that $\sigma(B)$ has a prime factor $p$ with $p>k r$ and $\operatorname{gcd}(p, B)=$ 1 by the definition of $A$. Let $p_{1}$ be the least such prime factor of $\sigma(B)$. Suppose $p_{1}^{e_{1}} \| A$, where $e_{1} \geq 1$. Then, if we put

$$
A^{\prime}:=A / p_{1}^{e_{1}} \quad \text { and } \quad B^{\prime}:=B p_{1}^{e_{1}},
$$

it is clear that (2.1)-(2.3) hold with $A^{\prime}$ and $B^{\prime}$ replacing $A$ and $B$. By the same argument as in [7], continuing the above procedure, we eventually obtain a factorisation

$$
A=p_{1}^{e_{1}} p_{2}^{e_{2}} \cdots p_{t}^{e_{t}}
$$

where $t=\omega(A)=\omega(N)-\omega(B) \leq r-1$. 
We note that the prime $p_{1}$ depends only on $B$, while for $i>1$, the prime $p_{i}$ depends only on $B$ and the exponents $e_{1}, \ldots, e_{i-1}$. It follows that for a given $B$, the cofactor $A$ (if $A>1$ ) is entirely determined by $e_{1}, \ldots, e_{t}$, and we have $e_{i} \leq \log _{5} x, i=1, \ldots, t$.

Let $B=q_{1}^{f_{1}} q_{2}^{f_{2}} \cdots q_{s}^{f_{s}}$. Then $f_{j} \leq \log _{3} x, j=1, \ldots, s, s+t=r$. Let $m$ be the number of odd primes not exceeding $k r$, so $m<k r / 2$. To estimate the number of possibilities for $B$ and $e_{1}, \ldots, e_{t}$, we first choose $s, 1 \leq s \leq r$, odd primes from the first $m$ odd primes, then choose positive integers $f_{j} \leq \log _{3} x, j=1, \ldots, s$, and nonnegative integers $e_{i} \leq \log _{5} x, i=1, \ldots, t$, with $s+t=r$ and obviously $e_{1}+\cdots+e_{t}+f_{1}+\cdots+$ $f_{s} \leq \log _{3} x$. The number of possibilities for $e_{1}+\cdots+e_{t}+f_{1}+\cdots+f_{s} \leq \log _{3} x$ is not larger than the number of nonnegative integer solutions of the equation

$$
e_{1}+\cdots+e_{t}+f_{1}+\cdots+f_{s}+y=\left\lfloor\log _{3} x\right\rfloor,
$$

which is $\left(\begin{array}{c}\left.\log _{3} x\right\rfloor+r \\ r\end{array}\right)$. It follows that the number of possibilities for $B$ and $e_{1}, \ldots, e_{t}$ is bounded by

$$
\left(\begin{array}{c}
\left\lfloor\log _{3} x\right\rfloor+r \\
r
\end{array}\right) \sum_{i=1}^{r}\left(\begin{array}{c}
m \\
i
\end{array}\right) \leq\left(\begin{array}{c}
\left\lfloor\log _{3} x\right\rfloor+r \\
r
\end{array}\right) \sum_{i=1}^{r}\left(\begin{array}{c}
\lfloor k r / 2\rfloor \\
i
\end{array}\right) .
$$

Recall Mertens' formula: for $x \geq 2$

$$
\prod_{p \leq x}\left(1-\frac{1}{p}\right)^{-1}=e^{\gamma} \log x+O(1)
$$

where $\gamma=0.577 \ldots$ is Euler's constant. Recall also the prime number theorem: if $p_{n}$ denotes the $n$th prime number, then $p_{n} \sim n \log n$. We have

$$
\begin{aligned}
k & =\frac{\sigma(N)}{N}<\prod_{p \mid N}\left(1+\frac{1}{p}+\frac{1}{p^{2}}+\cdots\right)=\prod_{p \mid N}\left(1-\frac{1}{p}\right)^{-1} \\
& \leq \frac{1}{2} \prod_{p \leq p_{r}}\left(1-\frac{1}{p}\right)^{-1} \sim \frac{e^{\gamma}}{2} \log r .
\end{aligned}
$$

By (1.1), we take $x=2^{4^{r}}$ so that the number of odd $k$-perfect numbers $n$ with $\omega(n) \leq r$ is bounded by

$$
\left(\begin{array}{c}
\left\lfloor 4^{r} \log _{3} 2\right\rfloor+r \\
r
\end{array}\right) \sum_{i=1}^{r}\left(\begin{array}{c}
\lfloor k r / 2\rfloor \\
i
\end{array}\right) \leq \frac{2^{k r / 2}}{r !}\left\lfloor 4^{r} \log _{3} 2+r\right\rfloor^{r} \leq \frac{2^{k r / 2}}{r !} 4^{r^{2}} .
$$

By (2.4) and the fact that the Taylor series for $\exp \left(2^{k / 2}\right)=\sum_{i=0}^{\infty} 2^{k i / 2} / i$ ! converges, $2^{k r / 2} / r$ ! must go to 0 as $r \rightarrow \infty$. This proves the theorem.

\section{Acknowledgement}

The author is pleased to thank the referee for valuable suggestions. He also thanks the referee for pointing out the reference [3] to him. 


\section{References}

[1] S. C. Chen and H. Luo, 'Bounds for odd k-perfect numbers', Bull. Aust. Math. Soc. 84(3) (2011), 475-480.

[2] R. J. Cook, 'Bounds for odd perfect numbers', in: Number Theory (Ottawa, ON, 1996), CRM Proceedings \& Lecture Notes, 19 (American Mathematical Society, Providence, RI, 1999), 67-71.

[3] L. X. Dai, H. Pan and C. Tang, 'Note on odd multiperfect numbers', Bull. Aust. Math. Soc., to appear.

[4] L. E. Dickson, 'Finiteness of the odd perfect and primitive abundant numbers with $\mathrm{n}$ distinct prime factors', Amer. J. Math. 35 (1913), 413-422.

[5] D. R. Heath-Brown, 'Odd perfect numbers', Math. Proc. Cambridge Philos. Soc. 115 (1994), 191-196.

[6] P. Nielsen, 'An upper bound for odd perfect numbers', Integers: Electronic J. Comb. Number Theory 3 (2003), A14, 9 pp. (electronic).

[7] P. Pollack, 'On Dickson's theorem concerning odd perfect numbers', Amer. Math. Monthly 118 (2011), 161-164.

[8] C. Pomerance, 'Multiply perfect numbers, Mersenne primes and effective computability', Math. Ann. 226 (1977), 195-206.

[9] E. Wirsing, 'Bemerkung zu der Arbeit über vollkommene Zahlen', Math. Ann. 137 (1959), $316-318$.

PINGZHI YUAN, School of Mathematics, South China Normal University, Guangzhou 510631, PR China

e-mail: yuanpz@scnu.edu.cn 